\title{
A new construct for the anti conformational locking of nucleosides: the dioxomethine transglycosidic tether ${ }^{\dagger}$
}

\author{
Michael P. Groziak ${ }^{* a}$ and Ronghui Lin ${ }^{b}$ \\ ${ }^{a}$ Pharmaceutical Discovery Division, SRI International 333 Ravenswood Avenue, Menlo Park, \\ CA 94025-3493, USA E-mail: michael.groziak@sri.com \\ ${ }^{b}$ Drug Discovery Research, R. W. Johnson Pharmaceutical Research Institute 920 U.S. Route \\ 202, P.O. Box 300, Raritan, NJ 08869-0602, USA E-mail: rlin@prius.jnj.com
}

(received 15 Sep 99; accepted 13 Feb 00; published on the web 21 Feb 00)

\begin{abstract}
Two members of a new class of anti conformationally locked 2'-deoxynucleoside mimics were synthesized starting from uridine through key 1-( $\beta$-D-arabinofuranosyl)uracil-6-carboxaldehyde intermediates. O5'-Mesylation of 1-( $\beta$-D-arabinofuranosyl)uracil-6-carboxaldehyde $(5 \mathrm{c})$ followed by pyridine-mediated transglycosidic displacement by the dominant 7,O2'-cyclic hemiacetal gave the locked 2',5'-dideoxyuridine mimic (7). Transglycosidic transacetalation in the 7,O2'-cyclic hemiacetal of 1-(5-deoxy-5,5-dimethoxy- $\beta$-D-arabinofuranosyl)uracil-6carboxaldehyde (18) gave the locked O5'-methyl-2'-deoxyuridine mimic (6b). These transglycosidic dioxomethine tether constructions involve proximity-assisted displacement reactions and provide an entry into new, highly biomimetic, anti conformationally locked 2'deoxynucleoside mimics for use as probes of conformation-activity relationships.
\end{abstract}

Keywords: Transglycosidic displacement, dioxomethine tether, 2'-deoxynucleoside mimics

\section{Introduction}

Conformational restriction is a very useful tool for studying the relationship between molecular topography and biochemical or medicinal activity of nucleosides. ${ }^{1}$ Transglycosidically tethered analogs are particularly useful when the focus of attention is the glycosidic bond, but in most cases the rotation of this bond is incompletely restricted-some residual rotational flexibility remains. The C6-spiro-fused 5,6-dihydrouridines $1\left(\mathrm{X}, \mathrm{Y}=\mathrm{F}, \mathrm{Cl}, \mathrm{Br}, \mathrm{NO}_{2}\right.$ ) reported by Honjo are a rare exception. ${ }^{2}$ They are 5,6-dihydrouridine arabinofuranosides in which both the $2^{\prime}$ and $5^{\prime}$ hydroxyl group oxygens have been attached to the pyrimidine C6 position. They are, in essence, completely rigidified mimics of the corresponding 2',5'-dideoxy-5,6-dihydrouridines (2). The cage-like carbohydrate moiety in 1 is related in a near-enantiomeric sense to 1,2,5-Obenzylidyne- $\beta$-L-arabinofuranose orthoesters reported by Kochetkov ${ }^{3}$ and to 1,2,5-O-ethylidyne$\alpha$-D-galactofuranose orthoesters reported by Bertolini and Glaudemans. ${ }^{4}$

The structural differences between 1 and its closest natural counterparts in RNA and DNA (dihydrouridine 3 and thymidine 4, respectively) limit their utility as probes of conformation/activity relationships. ${ }^{5}$ Nevertheless, the idea of transforming arabinofuranosides into conformationally locked 2'-deoxyribonucleoside mimics by constructing a tether over the 
$\beta$ face of the furanose ring has merit and would be particularly valuable if a high degree of biomimicry could be maintained by minimizing structural disturbances.

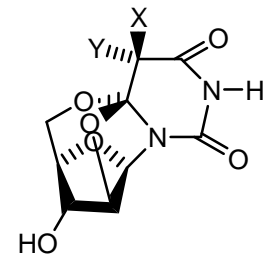

1

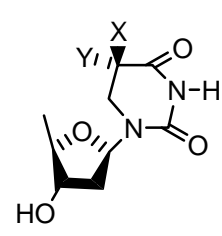

2

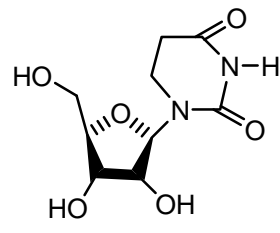

3

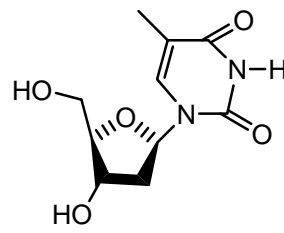

4

Some of the findings from our previous investigations ${ }^{6}$ into the chemistry of 6 -formyluridine, 6-formyl-2'-deoxyuridine, 6-formyl-1-( $\beta$-D-arabinofuranosyl)uracil, and 6-formylthymidine (5a$\mathrm{d}$, respectively) convinced us that this could be accomplished. The structures displayed by these compounds are dictated by their highly electrophilic carboxaldehyde group. While the ribonucleosides 5a,b and d exist as a solvent-sensitive collection of open (hydroxy-aldehyde) and 7,O5'-cyclic hemiacetal solution structures, the arabinofuranoside $5 \mathrm{c}$ exists exclusively as the 7,O2'-cyclic hemiacetal in all solvents examined. When taken together with the fact that 5'carboxaldehyde nucleoside derivatives are known to be susceptible to hydrate and hemiacetal formation, ${ }^{7}$ this raised the intriguing possibility that the $5^{\prime}$-carboxaldehyde derivative of $5 \mathrm{c}$ might exist at least partly in transglycosidically tethered form (6a). This form would be a highly biomimetic, anti conformationally locked and yet fully $O$-functionalizable 2'deoxyribonucleoside mimic. As the nucleoside or even the nucleotide (i.e., 6, $\mathrm{R}=\mathrm{PO}_{3} \mathrm{H}_{2}$ ), it would be a valuable bioprobe of conformation-activity relationships by virtue of an exceptionally close structural resemblance to naturally occurring counterparts. The anticipation of access not only to separate $\mathrm{C} 5^{\prime}$ epimeric versions but also to purine-based versions (from 8-formyl purine nucleosides ${ }^{8}$ ) only enhanced our interest in this attractive design construct, and so we began working on the construction of dioxomethine-tethered nucleosides.

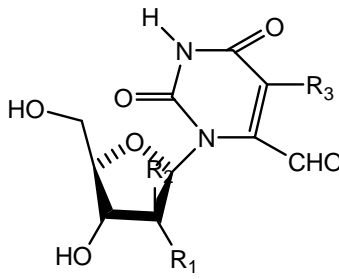

5a, $\mathrm{R}_{1}=\mathrm{OH}, \mathrm{R}_{2}=\mathrm{R}_{3}=\mathrm{H}$

b, $\mathrm{R}_{1}=\mathrm{R}_{2}=\mathrm{R}_{3}=\mathrm{H}$

c, $\mathrm{R}_{1}=\mathrm{R}_{3}=\mathrm{H}, \mathrm{R}_{2}=\mathrm{OH}$

d, $\mathrm{R}_{1}=\mathrm{R}_{2}=\mathrm{H}, \mathrm{R}_{3}=\mathrm{Me}$

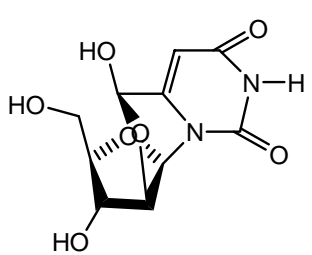

5c: 7,O2'-cyclic hemiacetal form

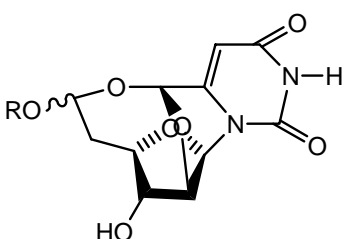

6a, $\mathrm{R}=\mathrm{H}$

b, $\mathrm{R}=\mathrm{Me}$

\section{Results and Discussion}

We searched for conditions that would assemble the desired dioxomethine tether, and first examined the ease of intramolecular dehydration in 5c to give a cyclic acetal. Even when heated at $65{ }^{\circ} \mathrm{C}, 5 \mathrm{c}$ was inert to exposure to $\mathrm{TsOH}$ or $\mathrm{TfOH}$ in $\left(\mathrm{CH}_{3}\right)_{2} \mathrm{SO}$ solution. The fact that the expected dioxomethine-tethered 2',5'-dideoxyuridine mimic 7 did not form under these conditions reflects the known difficulty of acetal formation in electron-deficient carbonyl groups, ${ }^{9}$ seen before for 5. ${ }^{6, \mathrm{c}}$ Conversion of the 5 '- or hemiacetal hydroxyl group in $5 \mathrm{c}$ to a good leaving group for intramolecular displacement was explored next. Treatment of 5c with an 
equivalent of $\mathrm{MsCl}$ in pyridine at increasingly elevated temperatures eventually produced 7 , which was isolated in a $43 \%$ yield. Under different conditions, exposure of $5 \mathrm{c}$ to $(\mathrm{EtO})_{3} \mathrm{CH}$ in the presence of $\mathrm{TfOH}$ gave 7 in a $57 \%$ yield.
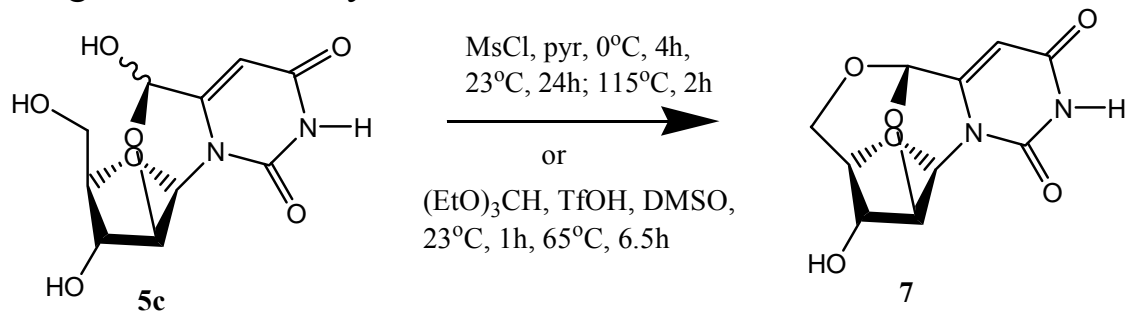

The dioxomethine-tethered 7 featuring a five-, a six-, a seven, and an eight-membered carbohydrate-associated ring was characterized by ${ }^{1} \mathrm{H},{ }^{13} \mathrm{C}$, COSY, and short-range ${ }^{1} \mathrm{H}-{ }^{13} \mathrm{C}$ HETCOR NMR, and both low- and high-resolution FAB mass spectral analyses. The ${ }^{1} \mathrm{H}$ NMR spectrum was especially diagnostic. Both the large $(14.2 \mathrm{~Hz})$ geminal coupling of the diastereotopic 5'- $\mathrm{CH}_{2}$ hydrogens and the moderate $(6.6 \mathrm{~Hz})$ vicinal one between $\mathrm{H} 1^{\prime}$ and $\mathrm{H} 2$ ' are consistent with the severe conformational restriction caused by the tether. A similar coupling constant pattern was found in the ${ }^{1} \mathrm{H}$ NMR spectrum of 2',3'-O-(isopropylidene)orotidine 5'lactone, a reference compound we prepared from 2',3'-O-isopropylidenated 5a. In addition, only one of the H5' resonances (the downfield pro- $R$ ) is coupled $(5.2 \mathrm{~Hz})$ to the $\mathrm{H} 4$ ' one in 7 . This same pattern is displayed not only by 2',3'-O-(isopropylidene)orotidine 5'-lactone, but also by certain 6,O5'-methanouridines reported by others. ${ }^{1 \mathrm{k}}$ By an MM calculation (Figure 1), 7 has a $\chi$ value (O4'-C1'-N1-C2 dihedral) of $276^{\circ}$, placing the glycosidic torsion in the "high" anti range.

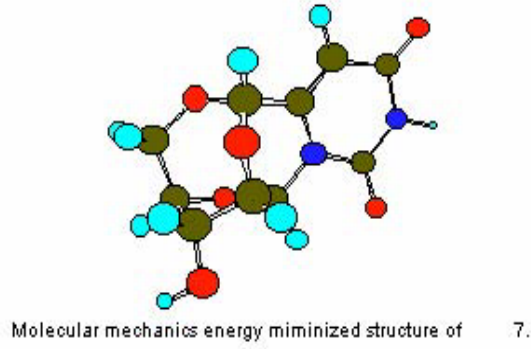

The task of preparing a nucleoside like 7 but with an additional oxygen functionality at C5' was addressed next. Deprotection of 2',3'-O-isopropylidene-6-formyluridine 5'-carboxaldehyde ${ }^{13}$ gave dialdehyde 8 (Scheme 1), but we were unable to generate its 2,2'-anhydride with $(\mathrm{PhO})_{2} \mathrm{CO}$, and thus could not access the arabinofuranoside. As shown in Scheme 1, only the 5'carboxaldehyde of 8 could be acetalated under standard conditions $(60 \%)$, and unfortunately a dehydration with $(\mathrm{PhO})_{2} \mathrm{CO}$ intended to give the 2,2'-anhydride gave instead a complex mixture from which the epoxy acetal 10 was isolated as the major product. ${ }^{10}$ The multistep pathway from 9 to 10 apparently involves a hydride transfer from $\mathrm{C} 1$ ' to the carboxaldehyde at some point.

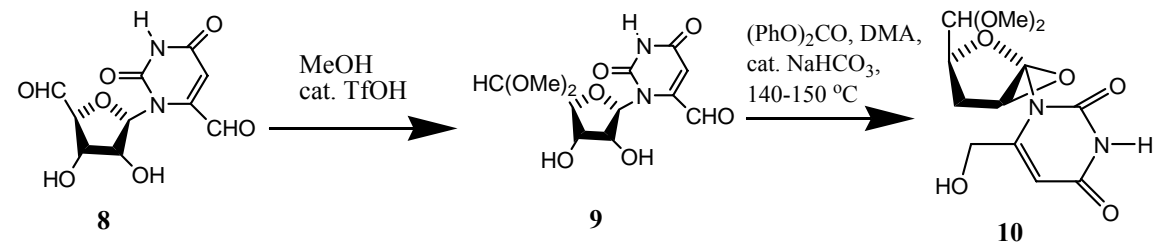

Scheme 1 
As shown in Scheme 2, the simultaneous masking of both carboxaldehyde groups in 8 as 1,3diphenylimidazolidin-2-yls (DPIs) gave $11(40 \%)$, but the reaction was sluggish and actually produced the 6-DPI 5'-dimethyl acetal (14, Scheme 3$)$ to a greater extent (47\%).

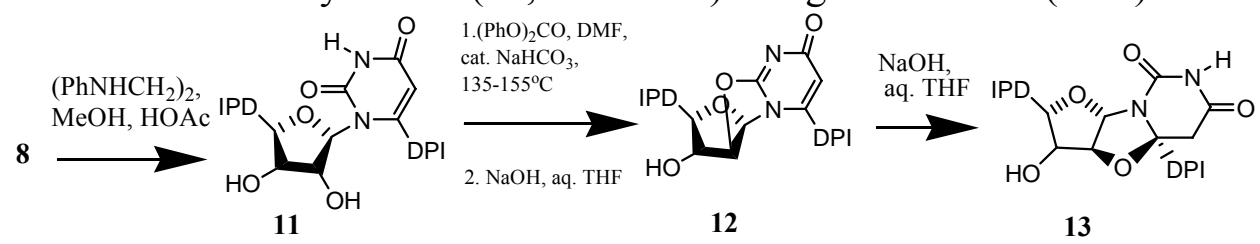

Scheme 2

Our first attempt to convert 11 to its 2,2'-anhydride employed 1.3 equivalents of $(\mathrm{PhO})_{2} \mathrm{CO}$. This did give some of the desired 12, but the yield was very low (7\%) and the O3'phenoxycarbonylated derivative and a substantial amount of starting material were isolated. In the second attempt, 3.5 equivalents of $(\mathrm{PhO})_{2} \mathrm{CO}$ and a slightly higher temperature were used to give the O3'-phenoxycarbonyl derivative of 12 in a satisfactory yield (66\%). Rapid saponification $\left(\mathrm{NaOH}, \mathrm{THF}, \mathrm{H}_{2} \mathrm{O}, 23{ }^{\circ} \mathrm{C}, 1 \mathrm{~h}\right)$ of this gave the desired $12(77 \%)$. Longer reaction times produced inseparable mixtures of 12 and the 6,O2'-cyclonucleoside 13, a compound generated by proximity-assisted intramolecular conjugate addition reaction in the open arabinofuranoside. Nucleoside 12 was treated with pTsOH in $\mathrm{Me}_{2} \mathrm{CO} / \mathrm{CH}_{2} \mathrm{Cl}_{2}$ in an attempt to remove both DPI groups prior to opening the 2,2'-anhydro linkage, but this failed.

The same 6-DPI 5'-dimethyl acetal 14 obtained previously from 8 was also obtained from 9, but in a better yield (86\%). As shown in Scheme 3, 2,2'-anhydro formation in 14 gave 15 (31\%) together with its O3'-phenoxycarbonylated derivative (58\%). Saponification of either of these gave the same 6,O2'-cyclonucleoside 16. Selective deprotection of 16 gave the hydrate 17 in a quantitative yield. Facile hydration in the corresponding aldehyde can be attributed to its two electronegative $\alpha$-heteroatoms. In a transformation critically important to the construction of the dioxomethine tether, hydrate 17 afforded hemiacetal 18 (quantitative) simply upon desiccation. Just as had been found for $5 \mathrm{c},{ }^{6 \mathrm{~b}, \mathrm{c}} 18$ exists as a $3: 1$ mixture of 7,O2'-cyclic hemiacetal diastereomers. Acid-catalyzed hydrolysis of the 5'-dimethyl acetal in 18 was sluggish, but eventually proceeded to give not the desired 6a, but its open 7, O2'-cyclic hemiacetal instead $(60 \%, 2: 1$ mixture of diastereomers).
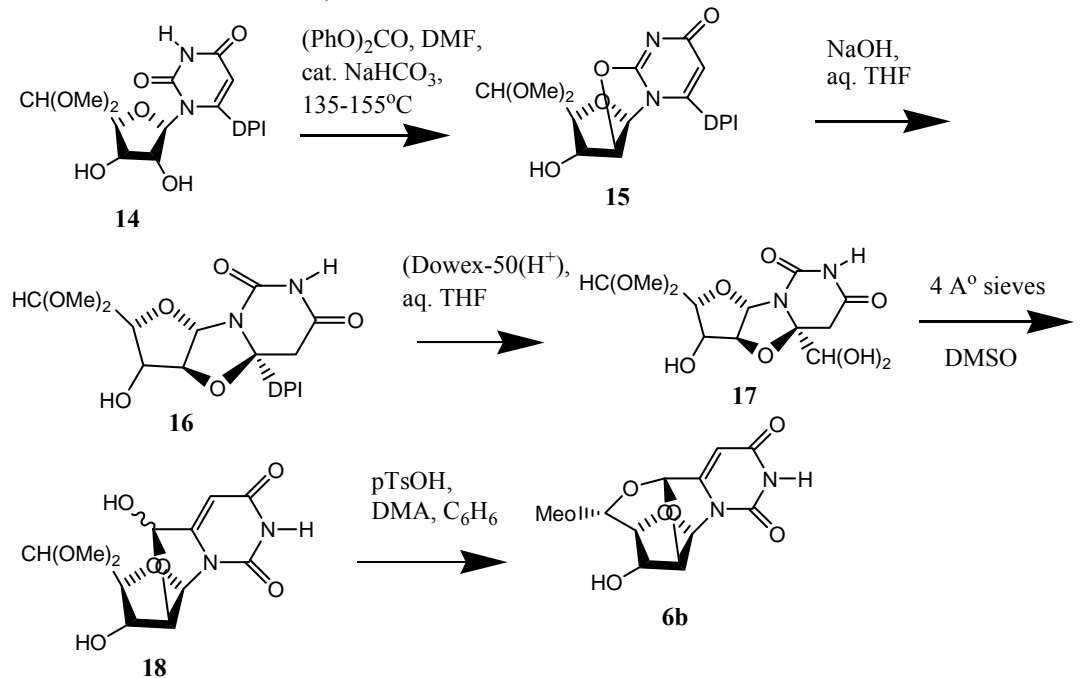

Scheme 3 
Attempts to close this to $6 \mathrm{a}$ by heating $\left(\mathrm{CH}_{3}\right)_{2} \mathrm{SO}$ containing $4 \AA$ molecular sieves, in $\mathrm{C}_{6} \mathrm{H}_{6} /$ DMA containing PPTS, in HMDS containing $\left(\mathrm{NH}_{4}\right)_{2} \mathrm{SO}_{4}$, or in pyridine containing $\mathrm{Ac}_{2} \mathrm{O}$ were unsuccessful. Similar attempts to access $6 \mathrm{~b}$ by heating 18 in $\left(\mathrm{CH}_{3}\right)_{2} \mathrm{SO}$ containing $\mathrm{TfOH}$ or $\mathrm{TsOH}$ also failed, but intramolecular acetal formation in 18 was promoted by the action of $\mathrm{TsOH}$ in $\mathrm{C}_{6} \mathrm{H}_{6} / \mathrm{DMA}$, and $6 \mathrm{~b}$ was isolated in a $25 \%$ yield. This dioxomethine-tethered $\mathrm{O}^{2}$-methylated 2'-deoxynucleoside mimic was characterized by ${ }^{1} \mathrm{H}$ and ${ }^{13} \mathrm{C}$ NMR and low- and high-resolution mass spectral analyses. Interestingly, it was isolated as a single diastereomer tentatively assigned the $5^{\prime}-R$ configuration because the ${ }^{3} \mathrm{~J} 4^{\prime}-5^{\prime}$ value $(3.8 \mathrm{~Hz})$ is close to the non-zero $(5.2 \mathrm{~Hz})^{3} \mathrm{~J} 4^{\prime}-5^{\prime}-$ pro- $R$ one observed in 7 . The fate of the absent 5 '-S diastereomer will be investigated, as will other, milder methods for generating $6 \mathrm{a}$ and methods for generating the nucleotide $(6, \mathrm{R}=$ $\mathrm{PO}_{3} \mathrm{H}_{2}$ ) analogs.

\section{Conclusions}

Our synthesis of 7 and $6 \mathrm{~b}$ demonstrates how a $\beta$-facial dioxomethine tether can be constructed onto a natural $\beta$-ribonucleoside framework by the careful and deliberate management of proximity-assisted addition and displacement reactions in arabinofuranoside intermediates. The new anti conformationally locked nucleosides now accessible are highly biomimetic and should prove to be valuable bioprobes for the study of conformation-activity relationships involving 2'deoxynucleosides, 2'-deoxynucleotides, and related species.

\section{Experimental Section}

General Procedures. Melting points were determined on a Thomas-Hoover UniMelt capillary apparatus and are uncorrected. Radial preparative-layer chromatography was performed on a Chromatotron instrument (Harrison Research, Inc., Palo Alto, CA) using Merck silica gel60 $\mathrm{PF}_{254}$ as the adsorbent, flash column chromatography was performed using 230-400 mesh ASTM Merck silica gel-60, and TLC analyses were performed on Analtech $250 \mu \mathrm{m}$ silica gel GF Uniplates. Lyophilizations were conducted on a Labconco Lypho-Lock 4.5 liter bench-top freeze-dryer. ${ }^{1} \mathrm{H}$ and ${ }^{13} \mathrm{C}$ NMR spectra were recorded on a Varian VXR-300 (300 and $\left.75 \mathrm{MHz}\right)$ or VXR-500 (500 and $125 \mathrm{MHz}$ ) instrument using (CH3)4Si or 2,2-dimethyl-2-silapentane-5sulfonic acid, sodium salt (DSS) $\left(\delta=0.0\right.$ for $\left.{ }^{1} \mathrm{H}\right)$, and $\mathrm{CDCl}_{3}\left(\delta=77.0\right.$ for $\left.{ }^{13} \mathrm{C}\right),\left(\mathrm{CD}_{3}\right) 2 \mathrm{SO}(\delta=$ 39.5 for $\left.{ }^{13} \mathrm{C}\right)$, or 1,4 -dioxane $\left(\delta=66.5\right.$ for ${ }^{13} \mathrm{C}$ in $\left.\mathrm{D}_{2} \mathrm{O}\right)$ as internal reference. Short-range ${ }^{1} \mathrm{H}-{ }^{13} \mathrm{C}$ heteronuclear shift correlation (HETCOR) 2D NMR spectra were obtained on the VXR-300 instrument. Except where noted, the purity of compounds was shown to be $>95 \%$ by TLC and high-field ${ }^{1} \mathrm{H}$ NMR. BuLi, iPr2NH, $\mathrm{HCO}_{2} \mathrm{Et}$, $\left(\mathrm{PhNHCH}_{2}\right)$ 2, 2-iodobenzoic acid, $\mathrm{KBrO}_{3}, \mathrm{TsOH}$, $\mathrm{NaBH}_{4}, 2,2^{\prime}$-biquinoline, anhydrous $\mathrm{iBuOH}$, and $1 \mathrm{M}$ TBAF in THF solution were purchased from the Aldrich Chemical Co. TBDMS-Cl and TBDPS-Cl were obtained from Hüls America, Inc. The $\mathrm{BuLi}$ was titrated by the modified Watson-Eastham procedure. ${ }^{11} \mathrm{THF}$ and $\mathrm{Et}_{2} \mathrm{O}$ were dried by distillation from Na-benzophenone ketyl under argon. Pyridine and $\mathrm{iPr}_{2} \mathrm{NH}$ were dried by distillation from $\mathrm{CaH}_{2}$ under argon. The $\mathrm{HCO}_{2}$ Et was dried by distillation from $\mathrm{P}_{2} \mathrm{O}_{5}$ under argon. Dowex-50 $\left(\mathrm{H}^{+}\right)$was obtained from the Sigma Chemical Co., and before use was washed with $1 \mathrm{~N} \mathrm{HCl}$ and then rinsed with distilled water until $\mathrm{pH}$ neutral. The Dess-Martin periodinane reagent was prepared according to the literature procedure. ${ }^{12}$ Elemental microanalyses and mass 
spectral analyses were obtained from the University of Illinois. MM calculations were performed using Chem3D Pro v.5.0 from Cambridge Scientific Computing, Inc.

(7S)-1-( $\beta$-D-Arabinofuranosyl)-6-(dihydroxymethyl)-7,02':7,05'-dianhydrouracil

Method A. A solution of $5 \mathrm{c}^{6 \mathrm{~b}, \mathrm{c}}(30.0 \mathrm{mg}, 0.110 \mathrm{mmol})$ in $0.5 \mathrm{~mL}$ of anhydrous pyridine was treated with one equivalent of $\mathrm{MsCl}(0.2 \mathrm{~mL})$ and the solution was stirred at $0{ }^{\circ} \mathrm{C}$ for $4 \mathrm{~h}, 23{ }^{\circ} \mathrm{C}$ for $24 \mathrm{~h}$, and then was heated at reflux for $2 \mathrm{~h}$. The mixture was evaporated to dryness in vacuo and the residue was separated by ascending preparative chromatography on silica gel (15\% $\mathrm{MeOH} / \mathrm{CH}_{2} \mathrm{Cl}_{2}$ as eluent) to give $12.0 \mathrm{mg}(43 \%)$ of $7:{ }^{1} \mathrm{H} \mathrm{NMR}\left(\left(\mathrm{CD}_{3}\right)_{2} \mathrm{SO}\right) \delta 11.6(1 \mathrm{H}, \mathrm{bs}, \mathrm{NH}$, exchanges with $\left.\mathrm{D}_{2} \mathrm{O}\right), 6.34\left(1 \mathrm{H}, \mathrm{d}, \mathrm{H} 1\right.$ '), $5.92(1 \mathrm{H}, \mathrm{s}, \mathrm{H} 7), 5.79(1 \mathrm{H}, \mathrm{s}, \mathrm{H} 5), 5.67\left(1 \mathrm{H}, \mathrm{d}, 3^{\prime}-\mathrm{OH}\right.$, exchanges with $\left.\mathrm{D}_{2} \mathrm{O}\right), 4.67\left(1 \mathrm{H}, \mathrm{d}, \mathrm{H} 2^{\prime}\right), 4.41\left(1 \mathrm{H}, \mathrm{d}, \mathrm{H} 3^{\prime}\right), 4.25\left(1 \mathrm{H}, \mathrm{d}, \mathrm{H} 4{ }^{\prime}\right), 4.08(1 \mathrm{H}, \mathrm{d}$ of d,

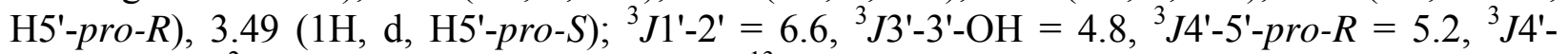
$5^{\prime}$ pro-S $=0,{ }^{2} J 5$ '-pro-R-5'-pro-S $=14.2 \mathrm{~Hz} .{ }^{13} \mathrm{C} \mathrm{NMR}\left(\left(\mathrm{CD}_{3}\right)_{2} \mathrm{SO}\right) \delta 163.0(\mathrm{C} 4), 150.8(\mathrm{C} 2)$, 146.4 (C6), 102.1 (C5), 91.3 (C7), 82.9 (C4'), 79.8 (C2'), 77.3 (C3'), 76.2 (C1'), 68.4 (C5'). Lowresolution FAB-mass spectrum, m/e $255.0\left(\mathrm{MH}^{+}\right)$. High-resolution FAB-mass spectrum for $\mathrm{C}_{10} \mathrm{H}_{11} \mathrm{~N}_{2} \mathrm{O}_{6}\left(\mathrm{MH}^{+}\right)$: calcd 255.0617, found 255.0618.

Method B. A solution of $5 \mathrm{c}^{6 \mathrm{~b}, \mathrm{c}}(15.0 \mathrm{mg}, 0.055 \mathrm{mmol})$ in $0.5 \mathrm{~mL}$ of $\left(\mathrm{CH}_{3}\right)_{2} \mathrm{SO}$ was treated with $50 \mu \mathrm{L}$ of TfOH and $0.2 \mathrm{~mL}$ (excess) of $(\mathrm{EtO}){ }_{3} \mathrm{CH}$. The reaction mixture was stirred first at $23{ }^{\circ} \mathrm{C}$ for $1 \mathrm{~h}$ and then at $65{ }^{\circ} \mathrm{C}$ for $6.5 \mathrm{~h}$, and then it was evaporated to dryness in vacuo. The residue was separated by the same procedure used in Method A to give $8.0 \mathrm{mg}(57 \%)$ of 7 .

2',3'-O-Isopropylideneorotidine 5'-lactone. A solution of 2',3'-O-isopropylidenated $5 \mathrm{a}^{6 \mathrm{c}}(312 \mathrm{mg}$, $1.0 \mathrm{mmol})$ and DCC $(0.8 \mathrm{~g}, 3.9 \mathrm{mmol})$ in $10 \mathrm{~mL}$ of anhydrous $\left(\mathrm{CH}_{3}\right)_{2} \mathrm{SO}$ was treated with anhydrous pyridine $(0.1 \mathrm{~mL})$ and TFA $(0.05 \mathrm{~mL})$, and the mixture was stirred at $23{ }^{\circ} \mathrm{C}$ for $50 \mathrm{~h}$. Water $(1 \mathrm{~mL})$ was added and the mixture was stirred for additional $0.5 \mathrm{~h}$. The dicyclohexylurea precipitate was removed by suction filtration, and the filtrate was evaporated to dryness at $50{ }^{\circ} \mathrm{C}$ in vacuo. Radial chromatography $\left(5 \% \mathrm{CH}_{3} \mathrm{OH} / \mathrm{CH}_{2} \mathrm{Cl}_{2}\right.$ as eluent) gave $161 \mathrm{mg}(52 \%)$ of the lactone as a white solid: $\operatorname{mp} 270-275^{\circ} \mathrm{C}$ (dec.). ${ }^{1} \mathrm{H}$ NMR $\left(\mathrm{CDCl}_{3}\right) \delta 8.32(1 \mathrm{H}, \mathrm{bs}, \mathrm{NH}), 6.08(1 \mathrm{H}$, s, H5), 5.98 (1H, d, H1'), 5.02 (1H, m, H5'), $4.97(1 \mathrm{H}, \mathrm{d}$ of d, H2'), 4.78 (1H, d, H3'), $4.72(1 \mathrm{H}$, m, H4'), $4.25\left(1 \mathrm{H}, \mathrm{m}, \mathrm{H} 5^{\prime}\right), 1.58\left(3 \mathrm{H}, \mathrm{s}, \mathrm{CH}_{3}\right), 1.37\left(3 \mathrm{H}, \mathrm{s}, \mathrm{CH}_{3}\right) .{ }^{1} \mathrm{H}$ NMR $\left(\left(\mathrm{CD}_{3}\right)_{2} \mathrm{SO}\right) \delta 11.74$ $(1 \mathrm{H}, \mathrm{bs}, \mathrm{NH}), 5.76(1 \mathrm{H}, \mathrm{s}, \mathrm{H} 5), 5.73\left(1 \mathrm{H}, \mathrm{d}, \mathrm{H} 1^{\prime}\right), 5.11\left(1 \mathrm{H}, \mathrm{d}, \mathrm{H} 5{ }^{\prime}\right), 4.68\left(1 \mathrm{H}, \mathrm{t}, \mathrm{H} 3{ }^{\prime}\right), 4.63(1 \mathrm{H}, \mathrm{t}$, H2'), 4.55 (d of d, 1H, H4'), $4.17(1 \mathrm{H}, \mathrm{t}, \mathrm{H} 5 "), 1.45\left(3 \mathrm{H}, \mathrm{s}, \mathrm{CH}_{3}\right), 1.28\left(3 \mathrm{H}, \mathrm{s}, \mathrm{CH}_{3}\right) .{ }^{13} \mathrm{C}$ NMR $\left(\left(\mathrm{CD}_{3}\right)_{2} \mathrm{SO}\right) \delta 165.5(\mathrm{C} 7), 162.8(\mathrm{C} 4), 148.7(\mathrm{C} 2), 143.1(\mathrm{C} 6), 112.0\left(\mathrm{C} 5\right.$ or $\left.C\left(\mathrm{CH}_{3}\right)_{2}\right), 103.0(\mathrm{C} 5$ or $\left.\mathrm{C}\left(\mathrm{CH}_{3}\right)_{2}\right), 95.0,86.4,85.4$, and 79.7 (each $\mathrm{Cl}^{\prime}, \mathrm{C}^{\prime}, \mathrm{C}^{\prime}$ ', or $\mathrm{C}^{\prime}$ '), 66.2 (C5'), 26.3 and 24.7 $\left(\mathrm{C}\left(\mathrm{CH}_{3}\right)_{2}\right)$. Low-resolution ACE-mass spectrum, m/e 310.2 $\left(\mathrm{M}^{+}\right), 311.2\left(\mathrm{MH}^{+}\right)$.

6-Formyluridine 5'-carboxaldehyde (8). A solution of 2',3'-O-isopropylidene-6-formyluridine $5^{\prime}$-carboxaldehyde ${ }^{13}(51.4 \mathrm{mg}, 0.17 \mathrm{mmol})$ in $50 \%$ aqueous TFA $(1 \mathrm{~mL})$ was stirred at $23{ }^{\circ} \mathrm{C}$ for $2 \mathrm{~h}$. The reaction mixture was evaporated to dryness, and residual TFA was removed from the residue by repetitive azeotropic coevaporation with water. Lyophilization afforded NMR-pure 8 in a quantitative yield: $210-220{ }^{\circ} \mathrm{C}$ (dec.). The NMR spectral features of 8 in $\mathrm{D}_{2} \mathrm{O}$ solution were consistent with dihydrate structure: ${ }^{1} \mathrm{H}$ NMR $\left(\mathrm{D}_{2} \mathrm{O}\right) \delta 6.14(\mathrm{~s}, 1 \mathrm{H}, \mathrm{H} 5), 5.98(\mathrm{~m}, 2 \mathrm{H}, \mathrm{H} 1$ ' and H7), 5.12 (d, 1H, H5'), 4.84 (s, H2' under HOD), 4.50 (pseudo-t, 1H, H3'), 3.80 (pseudo-t, 1H, H4'); ${ }^{3} J 1^{\prime}-2^{\prime}$ not well resolved, ${ }^{3} J 2^{\prime}-3^{\prime}=6.2,{ }^{3} J 3^{\prime}-4 '=5.9,{ }^{3} J 4^{\prime}-5^{\prime}=5.7 \mathrm{~Hz} .{ }^{13} \mathrm{C}$ NMR $\left(\mathrm{D}_{2} \mathrm{O}\right) \delta$ 165.7 (C4), 156.0 (C2), 151.6 (C6), 100.2 (C5), 92.1 (C1'), 89.9 (C5'), 85.8 (C7), 85.5 (C4'), 71.6 (C2'), 70.3 (C3'). UV $\lambda \max , \mathrm{nm}\left(\varepsilon^{\times} \times 10^{-3}\right)$ : ( $\left.\mathrm{H}_{2} \mathrm{O}\right) 261$ (8.9), 204 (9.2); (pH 1) 262 (7.8), 209 (7.2). Low-resolution CIMS, m/e $271.1\left(\mathrm{MH}^{+}\right)$; High-resolution CIMS calcd for $\mathrm{C}_{10} \mathrm{H}_{11} \mathrm{~N}_{2} \mathrm{O}_{7}\left(\mathrm{MH}^{+}\right)$: calcd 271.0566, found 271.0564. 
6-Formyluridine 5'-carboxaldehyde, 5'-dimethyl acetal (9). A suspension of 8 (2.00 g, $7.40 \mathrm{mmol})$ in $20 \mathrm{~mL}$ of absolute $\mathrm{MeOH}$ containing $\mathrm{TfOH}(100 \mu \mathrm{L})$ was heated at reflux under argon. After $20 \mathrm{~min}$, the mixture became homogeneous and 8 had been consumed, by TLC analysis. The solution was concentrated in vacuo and the residue was purified by radial chromatography $\left(10 \% \mathrm{MeOH} / \mathrm{CH}_{2} \mathrm{Cl}_{2}\right.$ as eluent) to give, after Abderhalden $\left(\mathrm{P}_{2} \mathrm{O}_{5}\right)$ drying, $1.40 \mathrm{~g}$ $(60 \%)$ of 9 as a yellow foam: ${ }^{1} \mathrm{H} \operatorname{NMR}\left(\mathrm{D}_{2} \mathrm{O}\right) \delta 6.12(1 \mathrm{H}, \mathrm{s}, \mathrm{H} 5), 5.96\left(1 \mathrm{H}, \mathrm{d}, \mathrm{H} 1{ }^{\prime}\right), 5.95(1 \mathrm{H}, \mathrm{s}$, hemiacetal $\mathrm{CH}), 4.81\left(1 \mathrm{H}, \mathrm{m}, \mathrm{H} 2^{\prime}\right), 4.62\left(1 \mathrm{H}, \mathrm{d}, \mathrm{H} 5^{\prime}\right), 4.54(1 \mathrm{H}$, pseudo-t, H3'), $3.89(1 \mathrm{H}$, pseudo-t, H4'), 3.51 and 3.44 (each $3 \mathrm{H}$, each s, each $\left.\mathrm{OCH}_{3}\right) ;{ }^{3} \mathrm{~J} 1^{\prime}-2^{\prime}=3.3,{ }^{3} \mathrm{~J}^{\prime}-4^{\prime}=6.6,{ }^{3} \mathrm{~J} 4^{\prime}-5^{\prime}=$ $6.9 \mathrm{~Hz} .{ }^{13} \mathrm{C}$ NMR $\left(\mathrm{D}_{2} \mathrm{O}\right) \delta 165.7(\mathrm{C} 4), 155.9$ and $151.4\left(\mathrm{C}_{2} / \mathrm{C} 6\right), 104.2\left(\mathrm{C} 5{ }^{\prime}\right), 100.2(\mathrm{C} 5), 92.4$ (C7), $85.8\left(\mathrm{C}^{\prime}\right), 82.2\left(\mathrm{C}^{\prime}\right), 72.0\left(\mathrm{C}^{\prime}\right), 70.3\left(\mathrm{C}^{\prime}\right), 55.3$ and $54.2\left(\right.$ two $\left.\mathrm{CH}_{3} \mathrm{O}\right)$.

1-(1-Dehydro-1-hydroxy-5-deoxy-5,5-dimethoxy-1,2-anhydro- $\alpha$-ID-arabinofuranosyl)-6hydroxymethyluracil (10). A solution of monoacetal $9(100.0 \mathrm{mg}, 0.316 \mathrm{mmol})$ and $(\mathrm{PhO})_{2} \mathrm{CO}$ (101.5 mg, $0.474 \mathrm{mmol})$ in $0.3 \mathrm{~mL}$ of anhydrous DMA was treated with $\mathrm{NaHCO}_{3}(2.0 \mathrm{mg})$ and then was heated at $140-150{ }^{\circ} \mathrm{C}$ for $15 \mathrm{~min}$. The solution was concentrated in vacuo, and the oily residue treated with $5 \mathrm{~mL}$ of anhydrous $\mathrm{Et}_{2} \mathrm{O}$. The resulting solid was collected, rinsed with additional anhydrous $\mathrm{Et}_{2} \mathrm{O}(3 \times 3 \mathrm{~mL})$, and then purified by ascending preparative chromatography $\left(10 \% \mathrm{MeOH} / \mathrm{CH}_{2} \mathrm{Cl}_{2}\right.$ as eluent) to give $26.6 \mathrm{mg}(27 \%)$ of $10:{ }^{1} \mathrm{H} \mathrm{NMR}\left(\mathrm{D}_{2} \mathrm{O}\right) \delta$ $5.74(1 \mathrm{H}, \mathrm{s}, \mathrm{H} 5), 5.49$ (1H, s, H2'), 4.94 and 4.74 (each 1H, each d, each H7), 4.55 (1H, d, H5'), $3.99\left(1 \mathrm{H}, \mathrm{d}, \mathrm{H} 3{ }^{\prime}\right), 3.95\left(1 \mathrm{H}, \mathrm{d}\right.$ of d, H4'), 3.48 and $3.42\left(\right.$ each $3 \mathrm{H}$, each s, each $\left.\mathrm{OCH}_{3}\right) ;{ }^{3} \mathrm{~J}^{\prime}{ }^{\prime}-3^{\prime}=0$, ${ }^{3} \mathrm{J3}^{\prime}-4^{\prime}=2.8,{ }^{3} \mathrm{~J}^{\prime}-5^{\prime}=7.3,{ }^{2} \mathrm{~J} 7 \mathrm{a}-7 \mathrm{~b}=16.8 \mathrm{~Hz} .{ }^{1} \mathrm{H} \mathrm{NMR}\left(\left(\mathrm{CD}_{3}\right)_{2} \mathrm{SO}\right) \delta 11.5(1 \mathrm{H}, \mathrm{bs}, \mathrm{NH}$, exchanges with $\left.\mathrm{D}_{2} \mathrm{O}\right), 7.3\left(1 \mathrm{H}, \mathrm{bs}, 7-\mathrm{OH}\right.$, exchanges with $\left.\mathrm{D}_{2} \mathrm{O}\right), 5.7\left(1 \mathrm{H}, \mathrm{d}, 3^{\prime}-\mathrm{OH}\right.$, exchanges with $\left.\mathrm{D}_{2} \mathrm{O}\right), 5.53(1 \mathrm{H}, \mathrm{s}, \mathrm{H} 5), 5.30\left(1 \mathrm{H}, \mathrm{s}, \mathrm{H} 2^{\prime}\right), 4.65(2 \mathrm{H}, \mathrm{q}, \mathrm{CH} 2), 4.28\left(1 \mathrm{H}, \mathrm{d}, \mathrm{H} 5^{\prime}\right), 4.71(1 \mathrm{H}, \mathrm{d}$ of d, H4'), $3.62\left(1 \mathrm{H}, \mathrm{d}\right.$ of d, H3'), 3.26 and 3.22 (each $3 \mathrm{H}$, each s, each $\left.\mathrm{OCH}_{3}\right) ;{ }^{3} \mathrm{~J}^{\prime} \mathrm{I}^{\prime}-3^{\prime}=0,{ }^{3} \mathrm{~J} 3^{\prime}-4^{\prime}$ $=2.2,{ }^{3} \mathrm{~J}^{\prime}-3^{\prime}-\mathrm{OH}=6.0,{ }^{3} \mathrm{~J} 4^{\prime}-5^{\prime}=7.7 \mathrm{~Hz} .{ }^{13} \mathrm{C} \mathrm{NMR}\left(\left(\mathrm{CD}_{3}\right)_{2} \mathrm{SO}\right) \delta 162.0(\mathrm{C} 4), 151.0$ and 148.0 (C2/C6), 103.1 (C5'), 99.0 (C1'), 96.7 (C5), 82.8 (C4'), 78.2 (C2'), 74.4 (C3'), $57.6\left(\mathrm{CH}_{2}\right), 54.0$ and 52.7 (two $\mathrm{CH}_{3} \mathrm{O}$ ). Low-resolution FAB-mass spectrum, m/e $317.1\left(\mathrm{MH}^{+}\right)$. High-resolution FAB-mass spectrum for $\mathrm{C}_{12} \mathrm{H}_{17} \mathrm{~N}_{2} \mathrm{O}_{8}\left(\mathrm{MH}^{+}\right)$: calcd 317.0985, found 317.0986.

5'-Deoxy-5',6-bis-(1,3-diphenylimidazolidin-2-yl)uridine (11) and 6-(1,3-diphenylimidazolidin-2-yl) uridine-5'-carbox-aldehyde, dimethyl acetal (14). A solution of crude 8 prepared from $200 \mathrm{mg}$ of its $2^{\prime}, 3^{\prime}-O$-isopropylidene derivative, ${ }^{11}\left(\mathrm{PhNHCH}_{2}\right)_{2}(303 \mathrm{mg}, 1.42 \mathrm{mmol})$, and $0.12 \mathrm{~mL}$ of glacial $\mathrm{AcOH}$ in $10 \mathrm{~mL}$ of absolute $\mathrm{CH}_{3} \mathrm{OH}$ was stirred for $3 \mathrm{~d}$ at $23{ }^{\circ} \mathrm{C}$. The reaction mixture was partitioned between saturated aqueous $\mathrm{NaHCO}_{3}$ and $\mathrm{CH}_{2} \mathrm{Cl}_{2}$, and the layers were separated and the aqueous phase was extracted with fresh $\mathrm{CH} 2 \mathrm{Cl} 2$. The organic solutions were combined, dried $\left(\mathrm{MgSO}_{4}\right)$, and then rotary evaporated to dryness. Column chromatography $\left(5 \% \mathrm{CH}_{3} \mathrm{OH} / \mathrm{CH}_{2} \mathrm{Cl}_{2}\right.$ as eluent) gave $168 \mathrm{mg}(40 \%)$ of 11 as a pink powder and $155 \mathrm{mg}(47 \%)$ of the 6-DPI, 5'dimethyl acetal derivative (14) as a yellowish foam. For 11: ${ }^{1} \mathrm{H} \mathrm{NMR}\left(\mathrm{CDCl}_{3}\right) \delta 9.6(1 \mathrm{H}$, bs, $\mathrm{NH}), 7.35-6.58\left(20 \mathrm{H}, \mathrm{m}\right.$, four $\left.\mathrm{C}_{6} \mathrm{H}_{5}\right) ; 5.90\left(1 \mathrm{H}, \mathrm{s}, \mathrm{H} 1^{\prime}\right), 5.78(1 \mathrm{H}, \mathrm{s}, \mathrm{H} 5), 5.68\left(1 \mathrm{H}, \mathrm{d}, \mathrm{H} 5^{\prime}\right), 5.46$ $(1 \mathrm{H}, \mathrm{s}, \mathrm{H} 7), 4.64-4.61\left(2 \mathrm{H}, \mathrm{m}, \mathrm{H} 2^{\prime}\right.$ and $\left.\mathrm{H} 3^{\prime}\right), 3.90\left(1 \mathrm{H}, \mathrm{d}\right.$ of d, H4'), 3.72-3.25 $\left(10 \mathrm{H}, \mathrm{m}, 2^{\prime}-\mathrm{OH}\right.$, $3^{\prime}-\mathrm{OH}$, and two $\left.\mathrm{NCH}_{2} \mathrm{CH}_{2} \mathrm{~N}\right) ;{ }^{3} \mathrm{~J}^{\prime}-2^{\prime}=0,{ }^{3} \mathrm{~J} 3^{\prime}-4^{\prime}=7.2,{ }^{3} \mathrm{~J} 4^{\prime}-5^{\prime}=1.9 \mathrm{~Hz} .{ }^{13} \mathrm{C}$ NMR $\left(\mathrm{CDCl}_{3}\right) \delta$ 163.0 (C4), 153.4, 150.8, 147.6, 146.4, 146.3, and 145.2 (C2, C6, and four $\left.\mathrm{C}_{6} \mathrm{H}_{5}\right), 129.5,129.4$, 129.1, 128.9, 122.5, 119.6, 118.3, 117.6, 117.4, 114.3, 113.5 and 113.0 (four $\mathrm{C}_{6} \mathrm{H}_{5}$ ), 102.7 (C5), $91.7(\mathrm{C} 7), 83.3\left(\mathrm{C}^{\prime}\right), 75.6\left(\mathrm{C}^{\prime}\right), 73.3\left(\mathrm{C}^{\prime}\right), 72.1$ and $69.7\left(\mathrm{C}^{\prime} / \mathrm{C}^{\prime}\right), 50.8,47.0,46.8$ and 46.1 (two $\left.\mathrm{NCH}_{2} \mathrm{CH}_{2} \mathrm{~N}\right)$. Low-resolution CI-mass spectrum, m/e $335.2(80 \%, \mathrm{~B}+1), 306.2\left(30 \%\right.$, $\mathrm{M}^{+}-$ $\left.\mathrm{B}-\mathrm{H}_{2} \mathrm{O}\right), 223.1$ (100\%). Low-resolution EI-mass spectrum, m/e $334.1\left(15 \%, \mathrm{~B}^{+}\right), 306.1(10 \%$, $\left.\mathrm{M}^{+}-\mathrm{B}-\mathrm{H}_{2} \mathrm{O}\right), 223.1$ (100\%). Low-resolution FAB-mass spectrum, $m / e 659.2\left(80 \%, \mathrm{MH}^{+}\right) .14:{ }^{1} \mathrm{H}$ $\operatorname{NMR}\left(\mathrm{CDCl}_{3}\right) \delta 10.2(1 \mathrm{H}, \mathrm{bs}, \mathrm{NH}), 7.28-6.69\left(10 \mathrm{H}, \mathrm{m}\right.$, two $\left.\mathrm{C}_{6} \mathrm{H}_{5}\right), 5.90(1 \mathrm{H}, \mathrm{s}, \mathrm{H} 5), 5.82(1 \mathrm{H}, \mathrm{s}$, 
H7), $5.62\left(1 \mathrm{H}, \mathrm{d}, \mathrm{H} 1^{\prime}\right), 4.75$ (1H, d of d, H2'), 4.53-4.49 (2H, m, H3' and H5'), 3.64 (1H, pseudot, H4'), 3.61-3.40 (6H, m, $\mathrm{NCH}_{2} \mathrm{CH}_{2} \mathrm{~N}, 2^{\prime}-\mathrm{OH}$, and 3'-OH), 3.39 and 3.37 (each $3 \mathrm{H}$, each s, each $\left.\mathrm{CH}_{3} \mathrm{O}\right) ;{ }^{3} \mathrm{~J} 1^{\prime}-2^{\prime}=2.4,{ }^{3} \mathrm{~J}^{\prime}-3^{\prime}=6.3,{ }^{3} \mathrm{~J} 4^{\prime}-5^{\prime}=7.2 \mathrm{~Hz} .{ }^{13} \mathrm{C} \mathrm{NMR}\left(\mathrm{CDCl}_{3}\right) \delta 63.2(\mathrm{C} 4), 153.4,151.4$, 147.3, 145.7 (C2, C6, and two $\mathrm{C}_{6} \mathrm{H}_{5}$ ), 129.5, 129.4, 122.2, 120.3, 118.1 and 115.3 (two $\mathrm{C}_{6} \mathrm{H}_{5}$ ), 104.0 (C5'), 102.5 (C5), 92.3 (C7), 82.1 (C4'), 75.9 (C1'), 72.5 and 70.5 (C2'/C3'), 54.7 and 53.4 (two $\left.\mathrm{CH}_{3} \mathrm{O}\right), 50.5$ and $47.5\left(\mathrm{NCH}_{2} \mathrm{CH}_{2} \mathrm{~N}\right)$. Low-resolution CI-mass spectrum, m/e $511.3(25 \%$, $\left.\mathrm{MH}^{+}\right), 479.3\left(40 \%, \mathrm{MH}^{+}-\mathrm{CH}_{3} \mathrm{OH}\right), 447.3\left(40 \%, \mathrm{MH}^{+}-2 \mathrm{CH}_{3} \mathrm{OH}\right), 223.2$ (100\%). Low-resolution EI-mass spectrum, m/e $510.4\left(15 \%, \mathrm{M}^{+}\right), 446.3\left(30 \%, \mathrm{M}^{+}-2 \mathrm{CH}_{3} \mathrm{OH}\right), 223.2$ (100\%). Highresolution CI-mass spectrum for $\mathrm{C}_{26} \mathrm{H}_{31} \mathrm{~N}_{4} \mathrm{O}_{7}\left(\mathrm{MH}^{+}\right)$: calcd 511.2192, found 511.2170.

5'-Deoxy-5',6-bis-(1,3-diphenylimidazolidin-2-yl)-2,2'-anhydrouridine (12). A solution of 11 $(110.0 \mathrm{mg}, 0.167 \mathrm{mmol})$ and $(\mathrm{PhO})_{2} \mathrm{CO}(46 \mathrm{mg}, 0.215 \mathrm{mmol})$ in $0.3 \mathrm{~mL}$ of anhydrous DMF was treated with $1 \mathrm{mg}$ of $\mathrm{NaHCO}_{3}$ and was heated at $110-130{ }^{\circ} \mathrm{C}$ for $0.5 \mathrm{~h}$ and then concentrated to dryness in vacuo. The mixture was then separated by ascending preparative chromatography using $5 \% \mathrm{MeOH} / \mathrm{CH}_{2} \mathrm{Cl}_{2}$ as eluent to give $12(8.0 \mathrm{mg}, 7 \%$ ) and its 3'-phenyl carbonate (25.2 $\mathrm{mg}$, $20 \%$ ). Treatment of $11(80 \mathrm{mg}, 0.121 \mathrm{mmol})$ with excess $(\mathrm{PhO})_{2} \mathrm{CO}(92 \mathrm{mg}, 0.429 \mathrm{mmol})$, additional $\mathrm{NaHCO}_{3}(2.0 \mathrm{mg})$, and at $135-155{ }^{\circ} \mathrm{C}$ in this procedure gave the $\mathrm{O}^{\prime}$ phenoxycarbonylated derivative of 12 in a $66 \%$ yield. $12:{ }^{1} \mathrm{H}$ NMR $\left(\mathrm{CDCl}_{3}\right) \delta 7.35-6.55(20 \mathrm{H}$, m, four C6H5), 6.10 (1H, d, H1'), $6.06(1 \mathrm{H}, \mathrm{s}, \mathrm{H} 5), 6.03(1 \mathrm{H}, \mathrm{s}, \mathrm{H} 7), 5.84$ (1H, s, 3'-OH), 5.53 $\left(1 \mathrm{H}, \mathrm{s}, \mathrm{H} 5^{\prime}\right), 4.89$ (1H, d of d, H2'), 4.52 (1H, m, H3'), $4.25\left(1 \mathrm{H}, \mathrm{d}, \mathrm{H} 4{ }^{\prime}\right), 3.80-3.32$ (8H, m, two $\left.\mathrm{NCH}_{2} \mathrm{CH}_{2} \mathrm{~N}\right) ;{ }^{3} J 1^{\prime}-2^{\prime}=5.7,{ }^{3} \mathrm{~J}^{\prime}-3^{\prime}=2.1 \mathrm{~Hz},{ }^{3} \mathrm{~J} 3^{\prime}-4^{\prime}=6.6 .{ }^{13} \mathrm{C} \mathrm{NMR}^{\prime}\left(\mathrm{CDCl}_{3}\right) \delta 172.4(\mathrm{C} 4), 160.7$ (C2), 148.0, 147.2, 146.5, 145.8, 144.8 (C6 and four $\left.\mathrm{C}_{6} \mathrm{H}_{5}\right), 129.7,129.6,129.4,129.2,122.5$, 118.8, 118.2, 118.1, 117.8, 113.4, 113.3, and 112.8 (two $\left.\mathrm{C}_{6} \mathrm{H}_{5}\right), 108.1$ (C5), $89.4\left(\mathrm{C}^{\prime}\right), 86.7$ (C1'), 85.7 (C4'), 74.9 (C3'), 73.8 (C7), 73.5 (C5'), 50.8, 47.0, 46.9 and 45.6 (two $\mathrm{NCH}_{2} \mathrm{CH}_{2} \mathrm{~N}$ ). Low-resolution FAB-mass spectrum, $m / e$ $641.3\left(70 \%, \mathrm{MH}^{+}\right), 223.2(100 \%)$. High-resolution FAB-mass spectrum for $\mathrm{C}_{38} \mathrm{H}_{37} \mathrm{~N}_{6} \mathrm{O}_{4}\left(\mathrm{MH}^{+}\right)$: calcd 641.2876, found 641.2887. O3'Phenoxycarbonylated 12: ${ }^{1} \mathrm{H}$ NMR $\left(\mathrm{CDCl}_{3}\right) \delta 7.45-6.62\left(25 \mathrm{H}, \mathrm{m}\right.$, five $\left.\mathrm{C}_{6} \mathrm{H}_{5}\right), 6.47\left(1 \mathrm{H}, \mathrm{d}, \mathrm{H} 1^{\prime}\right)$, $6.14(1 \mathrm{H}, \mathrm{s}, \mathrm{H} 5), 5.96$ (1H, s, H7), 5.79 (1H, d, H5'), 5.60 (1H, d, H3'), 5.27 (1H, d, H2'), 4.82 $\left(1 \mathrm{H}, \mathrm{d}\right.$ of d, H4'), 3.80-3.35 (8H, m, two $\left.\mathrm{NCH}_{2} \mathrm{CH}_{2} \mathrm{~N}\right) ;{ }^{3} J 1^{\prime}-2^{\prime}=5.4,{ }^{3} 3^{\prime}-4^{\prime}=2.7,{ }^{3} \mathrm{~J}^{\prime}-5^{\prime}=1.2$ Hz. ${ }^{13} \mathrm{C}$ NMR $\left(\mathrm{CDCl}_{3}\right) \delta 171.6(\mathrm{C} 4), 160.4(\mathrm{C} 2), 152.6,150.5,147.8,147.3,146.2,145.8,144.9$ $\left(\mathrm{C} 6, \mathrm{C}_{6} \mathrm{H}_{5} \mathrm{OCO}_{2}\right.$, and five $\left.\mathrm{C}_{6} \mathrm{H}_{5}\right), 129.8,129.7,129.6,129.5,129.4,126.7,122.5,120.6,119.6$, 119.3, 118.7, 117.8, 114.8, 113.6 and 113.2 (three $\mathrm{C}_{6} \mathrm{H}_{5}$ ), 109.6 (C5), $88.5\left(\mathrm{C}^{\prime}\right), 88.3\left(\mathrm{C} 1{ }^{\prime}\right), 85.8$ (C2'), 78.0 (C3'), 74.5 (C7), 73.4 (C5'), 50.7, 49.3, 47.6 and 46.0 (two $\mathrm{NCH}_{2} \mathrm{CH}_{2} \mathrm{~N}$ ). Lowresolution FAB-mass spectrum, $m / e 761.3\left(60 \%, \mathrm{MH}^{+}\right)$.

(6R)-6,O2'- Anhydro-1-[5-deoxy-5-(1,3-diphenylimidazolidin-2-yl)- $\beta$ ]-D-arabinofuranosyl]6-(1,3-diphenylimidazo-lidin-2-yl)-5,6-dihydrouracil (13). Saponification of $60 \mathrm{mg}$ $(0.079 \mathrm{mmol})$ of the O3'-phenoxycarbonylated 12 in a mixture of $0.123 \mathrm{~mL}$ of $1 \mathrm{M} \mathrm{NaOH}$, $0.9 \mathrm{~mL}$ of $\mathrm{H}_{2} \mathrm{O}$, and $1.4 \mathrm{~mL}$ of THF at $23{ }^{\circ} \mathrm{C}$ for $0.5 \mathrm{~h}$ gave, after chromatographic separation, $39.1 \mathrm{mg}(77 \%)$ of 12 . Saponification of $12 \mathrm{in} \mathrm{THF} / \mathrm{MeOH} / \mathrm{H}_{2} \mathrm{O}$ at $23{ }^{\circ} \mathrm{C}$ for $2.5 \mathrm{~h}$ afforded an inseparable mixture of 12 and the dihydrouracil 13 by ${ }^{1} \mathrm{H}$ NMR analysis. $13:{ }^{1} \mathrm{H} \mathrm{NMR}\left(\mathrm{CDCl}_{3}\right) \delta$ 2.94 and 2.29 (each $1 \mathrm{H}$, each d, H5a and H5b), $\left.2.8\left(1 \mathrm{H}, \mathrm{bs}, 3^{\prime}-\mathrm{OH}\right) ;{ }^{2} \mathrm{~J} 5 \mathrm{a}-5 \mathrm{~b}=15.9 \mathrm{~Hz}\right)$.

5'-Deoxy-5',5'-dimethoxy-6-(1,3-diphenylimidazolidin-2-yl)-2,2'-anhydrouridine (15). A solution of $14(120 \mathrm{mg}, 0.235 \mathrm{mmol}),(\mathrm{PhO})_{2} \mathrm{CO}(100.7 \mathrm{mg}, 0.47 \mathrm{mmol})$ and $\mathrm{NaHCO}_{3}(2.0 \mathrm{mg})$ in $0.5 \mathrm{~mL}$ of DMF was heated at $135-155{ }^{\circ} \mathrm{C}$ for $1 \mathrm{~h}$. The solution was rotary evaporated to dryness in vacuo, and the residue purified by chromatography to afford $15(35.8 \mathrm{mg}, 31 \%)$ and 3'-phenoxycarbonylated $15(83.7 \mathrm{mg}, 58 \%)$. 15: ${ }^{1} \mathrm{H}$ NMR $\left(\mathrm{CDCl}_{3}\right) \delta 7.32-6.66(10 \mathrm{H}, \mathrm{m}$, two $\left.\mathrm{C}_{6} \mathrm{H}_{5}\right), 6.53\left(1 \mathrm{H}, \mathrm{d}, \mathrm{H} 1^{\prime}\right), 6.23$ and 6.03 (each $1 \mathrm{H}$, each s, $\mathrm{H} 5$ and $\left.\mathrm{H} 7\right), 6.20\left(1 \mathrm{H}, \mathrm{bs}, 3{ }^{\prime}-\mathrm{OH}\right.$, 
exchanges with $\left.\mathrm{D}_{2} \mathrm{O}\right), 5.43\left(1 \mathrm{H}, \mathrm{d}, \mathrm{H} 2^{\prime}\right), 4.75\left(1 \mathrm{H}, \mathrm{s}, \mathrm{H} 3^{\prime}\right), 4.42$ and 4.24 (each $1 \mathrm{H}$, each d, $\left.\mathrm{H}^{\prime} / \mathrm{H}^{\prime}\right), 3.75-3.45\left(4 \mathrm{H}, \mathrm{m}, \mathrm{NCH}_{2} \mathrm{CH}_{2} \mathrm{~N}\right), 3.30\left(6 \mathrm{H}, \mathrm{s}\right.$, two $\left.\mathrm{CH}_{3} \mathrm{O}\right){ }^{3}{ }^{3} 1^{\prime}-2^{\prime}=6.3,{ }^{3} \mathrm{~J} 4^{\prime}-5^{\prime}=3.3 \mathrm{~Hz}$. ${ }^{13} \mathrm{C} \mathrm{NMR}\left(\mathrm{CDCl}_{3}\right) \delta 173.4(\mathrm{C} 4), 161.4(\mathrm{C} 2), 148.7,148.2$, and $144.5\left(\mathrm{C} 6\right.$ and two $\left.\mathrm{C}_{6} \mathrm{H}_{5}\right), 129.5$, 129.4, 122.8, 118.8, 118.1, and 112.4 (two $\mathrm{C}_{6} \mathrm{H}_{5}$ ), 107.3 and 103.3 (C5/C5'), 90.1, 88.9, 88.8, 76.5, and 73.1 (C1', C4', C2', C3', and C7), 56.2 and 55.9 (each $\mathrm{CH}_{3} \mathrm{O}$ ), 51.7 and 45.0 $\left(\mathrm{NCH}_{2} \mathrm{CH}_{2} \mathrm{~N}\right)$. Low-resolution FAB-mass spectrum, m/e 493.1 (35\%, $\left.\mathrm{MH}^{+}\right)$. High-resolution FAB-mass spectrum for $\mathrm{C}_{26} \mathrm{H}_{29} \mathrm{~N}_{4} \mathrm{O}_{6}\left(\mathrm{MH}^{+}\right)$: calcd 493.2087, found 493.2090.

O3'-Phenoxycarbonylated 15: ${ }^{1} \mathrm{H}$ NMR $\left(\mathrm{CDCl}_{3}\right.$.) $\delta$ 7.42-6.65 $\left(15 \mathrm{H}, \mathrm{m}\right.$, three $\left.\mathrm{C}_{6} \mathrm{H}_{5}\right), 6.66(1 \mathrm{H}, \mathrm{d}$, H1'), 6.27 and 6.12 (each 1H, each s, $\mathrm{H} 5$ and $\mathrm{H} 7), 5.42\left(1 \mathrm{H}, \mathrm{s}, \mathrm{H} 3^{\prime}\right), 5.33\left(1 \mathrm{H}, \mathrm{d}, \mathrm{H} 2{ }^{\prime}\right), 4.45(1 \mathrm{H}$, d, H4'), $4.25\left(1 \mathrm{H}, \mathrm{d}, \mathrm{H} 5^{\prime}\right), 3.75-3.35\left(8 \mathrm{H}, \mathrm{m}\right.$, two $\left.\mathrm{NCH}_{2} \mathrm{CH}_{2} \mathrm{~N}\right), 3.32\left(6 \mathrm{H}\right.$, s, two $\left.\mathrm{CH}_{3} \mathrm{O}\right) ;{ }^{3} J 1^{\prime}-2^{\prime}=$ $6.0,{ }^{3} \mathrm{~J}^{\prime}-5^{\prime}=3.0 \mathrm{~Hz} .{ }^{13} \mathrm{C} \mathrm{NMR}\left(\mathrm{CDCl}_{3}\right) \delta 173.2(\mathrm{C} 4), 161.1(\mathrm{C} 2), 152.4,150.5,148.9,147.8$, $144.6\left(\mathrm{C} 6, \mathrm{C}_{6} \mathrm{H}_{5} \mathrm{OCO}_{2}\right.$, and three $\left.\mathrm{C}_{6} \mathrm{H}_{5}\right), 129.6,129.5,129.3,126.5,123.0,120.5,118.2,115.4$, and 112.5 (three $\mathrm{C}_{6} \mathrm{H}_{5}$ ), 107.7 (C5), 102.9 (C5'), 89.9 (C1'), 86.0 (C4'), 85.1 (C3'), 81.4 (C2'), 73.1 (C7), 56.8 and 56.6 (each $\left.\mathrm{CH}_{3} \mathrm{O}\right), 52.2$ and $44.9\left(\mathrm{NCH}_{2} \mathrm{CH}_{2} \mathrm{~N}\right)$. Low-resolution FAB-mass spectrum, m/e $613.3\left(40 \%, \mathrm{MH}^{+}\right)$. High-resolution FAB-mass spectrum for $\mathrm{C}_{33} \mathrm{H}_{33} \mathrm{~N}_{4} \mathrm{O}_{8}\left(\mathrm{MH}^{+}\right)$: calcd 613.2298 , found 613.2288 .

(6R)-6,O2'-Anhydro-1-(5-deoxy-5,5-dimethoxy- $\beta$-D-arabinofuranosyl)-6-(1,3-diphenylimidazolidin-2-yl)-5,6-dihy-drouracil (16). Saponification of the O3'-phenoxycarbonylated 15 from above $(83.7 \mathrm{mg}, 0.136 \mathrm{mmol})$ in a mixture of $0.2 \mathrm{~mL}$ of $1 \mathrm{M} \mathrm{NaOH}$ and $2.0 \mathrm{~mL}$ of $50 \%$ aqueous THF at $23{ }^{\circ} \mathrm{C}$ overnight gave, after ascending chromatographic purification (silica gel, $5 \% \mathrm{MeOH} / \mathrm{CH}_{2} \mathrm{Cl}_{2}$ as eluent), the dihydrouridine $16(62.7 \mathrm{mg}, 90 \%)$. The intermediacy of $15 \mathrm{in}$ this transformation was verified by TLC. A similar saponification of 15 (25.0 mg, $0.136 \mathrm{mmol})$ for $24 \mathrm{~h}$ also gave $16(23.8 \mathrm{mg}, 92 \%)$ in a reaction which when halted after only $12 \mathrm{~h}$ was found to afford 1-(5-deoxy-5,5-dimethoxy- $\beta$-D-arabinofuranosyl)-6-(1,3-diphenylimidazolidin-2yl)uracil $(19 \%)$ in addition to $16(66 \%)$.

16: ${ }^{1} \mathrm{H}$ NMR $\left(\mathrm{CDCl}_{3}\right) \delta 7.5(1 \mathrm{H}, \mathrm{bs}, \mathrm{NH}), 7.25-6.70\left(10 \mathrm{H}, \mathrm{m}\right.$, two $\left.\mathrm{C}_{6} \mathrm{H}_{5}\right), 5.92\left(1 \mathrm{H}, \mathrm{d}, \mathrm{H} 1{ }^{\prime}\right), 5.71$ $(1 \mathrm{H}, \mathrm{s}, \mathrm{H} 7), 4.49$ (1H, d, H5'), 4.43 (1H, d of d, H2'), 4.29 (1H, d, H3'), 3.85-3.30 (5H, m, H4' and $\mathrm{NCH}_{2} \mathrm{CH}_{2} \mathrm{~N}$ ), 3.47 and 3.46 (each $3 \mathrm{H}$, each s, each $\mathrm{CH}_{3} \mathrm{O}$ ), 2.97 and 2.43 (each $1 \mathrm{H}$, each d, H5a and H5b), 2.5 (1H, bs, 3'-OH); ${ }^{3} \mathrm{~J} 5 \mathrm{a}-5 \mathrm{~b}=15.9,{ }^{3} \mathrm{~J} 1^{\prime}-2^{\prime}=4.2,{ }^{3} \mathrm{~J} 3^{\prime}-4^{\prime}=6.6,{ }^{3} \mathrm{~J} 4^{\prime}-5^{\prime}=4.5 \mathrm{~Hz}$. ${ }^{13} \mathrm{C} \mathrm{NMR}\left(\mathrm{CDCl}_{3}\right) \delta 165.7(\mathrm{C} 4), 150.1,147.8$ and $146.3\left(\mathrm{C} 2\right.$ and two $\left.\mathrm{C}_{6} \mathrm{H}_{5}\right), 129.1,128.9,119.8$, 119.0116 .3 and 114.1 (two $\mathrm{C}_{6} \mathrm{H}_{5}$ ), 104.1 (C5'), 100.2 (C6), 89.2 (C2'), 88.8 (C1'), 84.6 (C4'), $77.7(\mathrm{C} 7), 74.4(\mathrm{C} 3), 56.7$ and $55.1\left(\right.$ each $\left.\mathrm{CH}_{3} \mathrm{O}\right), 49.8$ and $48.4\left(\mathrm{NCH}_{2} \mathrm{CH}_{2} \mathrm{~N}\right), 38.5(\mathrm{C} 5)$. Lowresolution CI-mass spectrum, m/e $511.3\left(30 \%, \mathrm{MH}^{+}\right), 479.2\left(30 \%, \mathrm{MH}^{+}-\mathrm{MeOH}\right)$. Highresolution CI-mass spectrum for $\mathrm{C}_{26} \mathrm{H}_{31} \mathrm{~N}_{4} \mathrm{O}_{7}\left(\mathrm{MH}^{+}\right)$: calcd 511.2193, found 511.2183.

\section{1-(5-Deoxy-5,5-dimethoxy- $\beta$-D-arabinofuranosyl)-6-(1,3-diphenylimidazolidin-2-yl)uracil.}

${ }^{1} \mathrm{H}$ NMR $\left(\mathrm{CDCl}_{3}\right) \delta 9.0\left(1 \mathrm{H}\right.$, br, $\mathrm{NH}$, exchanges with $\left.\mathrm{D}_{2} \mathrm{O}\right), 7.32-6.50\left(10 \mathrm{H}, \mathrm{m}\right.$, two $\left.\mathrm{C}_{6} \mathrm{H}_{5}\right), 6.22$ $\left(1 \mathrm{H}, \mathrm{d}, \mathrm{H} 1^{\prime}\right), 6.00$ and 5.81 (each $1 \mathrm{H}$, each s, H7/H5), $4.56(2 \mathrm{H}$, pseudo-t, H5'/H2'), $4.48(1 \mathrm{H}, \mathrm{d}$, H2'), $4.29\left(1 \mathrm{H}, \mathrm{d}, \mathrm{H} 3^{\prime}\right), 3.75\left(1 \mathrm{H}, \mathrm{d}\right.$ of d, H4'), 3.58 and 3.27 (each $3 \mathrm{H}$, each m, $\mathrm{NCH}_{2} \mathrm{CH}_{2} \mathrm{~N}$ ), 3.44 and 3.40 (each $3 \mathrm{H}$, each s, each $\mathrm{CH}_{3} \mathrm{O}$ ), 2.6 and 1.8 (each $1 \mathrm{H}$, each br, 2'-OH/3'-OH, exchanges with $\left.\mathrm{D}_{2} \mathrm{O}\right) ;{ }^{3} \mathrm{~J} 5 \mathrm{a}-5 \mathrm{~b}=15.9,{ }^{3} \mathrm{~J} 1^{\prime}-2^{\prime}=4.2,{ }^{3} \mathrm{~J}^{\prime}-4^{\prime}=6.6,{ }^{3} \mathrm{~J} 4^{\prime}-5^{\prime}=4.5 \mathrm{~Hz} .{ }^{13} \mathrm{C}$ NMR $\left(\mathrm{CDCl}_{3}\right) \delta 162.4(\mathrm{C} 4), 150.9,150.8,147.8$ and $146.0\left(\mathrm{C} 2, \mathrm{C} 6\right.$, and two $\left.\mathrm{C}_{6} \mathrm{H}_{5}\right), 129.3,129.2$, 122.2, 119.3, 117.4 and 112.8 (two $\left.\mathrm{C}_{6} \mathrm{H}_{5}\right), 103.6$ and $100.7(\mathrm{C} 5 / \mathrm{C} 5$ '), 82.5, 81.2, 80.8, 76.8 and $76.0\left(\mathrm{C}^{\prime} / \mathrm{C}^{\prime} / \mathrm{C}^{\prime} / \mathrm{C}^{\prime} / \mathrm{C}^{\prime}\right), 56.6$ and 55.4 (each $\left.\mathrm{CH}_{3} \mathrm{O}\right), 47.5$ and $41.7\left(\mathrm{NCH}_{2} \mathrm{CH}_{2} \mathrm{~N}\right)$. Lowresolution FAB-mass spectrum, m/e $511.3\left(40 \%, \mathrm{MH}^{+}\right), 479.2$ (20\%, $\left.\mathrm{MH}^{+}-\mathrm{MeOH}\right)$. Highresolution FAB-mass spectrum for $\mathrm{C}_{26} \mathrm{H}_{31} \mathrm{~N}_{4} \mathrm{O}_{7}\left(\mathrm{MH}^{+}\right)$: calcd 511.2193, found 511.2199. 
(6R)-6,02'-Anhydro-1-(5-deoxy-5,5-dimethoxy- $\beta$-D-arabinofuranosyl)-6-(dihydroxymethyl)-5,6-dihydro-uracil (17). When an attempt at hydrolyzing the DPI group in 16 using $\mathrm{TsOH}$ in $\mathrm{Me}_{2} \mathrm{CO} / \mathrm{CH}_{2} \mathrm{Cl}_{2}$ gave a chromatographically inseparable mixture, this deprotection was instead effected by stirring a mixture of $16(32.9 \mathrm{mg}, 0.064 \mathrm{mmol})$ and Dowex50 (500 $\mathrm{mg}, \mathrm{H}^{+}$form) in $2.0 \mathrm{~mL}$ of $67 \%$ aqueous THF at $23{ }^{\circ} \mathrm{C}$ for $3 \mathrm{~d}$. Upon a removal of the resin and solvents, hydrate 17 was isolated $\left(21.5 \mathrm{mg}\right.$, quantitative). ${ }^{1} \mathrm{H}$ NMR $\left(\left(\mathrm{CD}_{3}\right)_{2} \mathrm{SO}\right) \delta 10.3(1 \mathrm{H}, \mathrm{bs}, \mathrm{NH}$, exchanges with $\left.\mathrm{D}_{2} \mathrm{O}\right), 6.45\left(1 \mathrm{H}, \mathrm{d}\right.$, hydrate $\mathrm{OH}$, exchanges with $\left.\mathrm{D}_{2} \mathrm{O}\right), 6.15(1 \mathrm{H}$, d, exchanges upon addition of $\mathrm{D}_{2} \mathrm{O}$, hydrate $\left.\mathrm{OH}\right), 5.89(1 \mathrm{H}, \mathrm{d}, \mathrm{H} 1$ '), $5.54(1 \mathrm{H}$, d, exchanges, 3'-OH), $4.75(1 \mathrm{H}$, pseudo-t, hydrate $\mathrm{CH}), 4.44$ (1H, d, H2'), 4.41 (1H, d, H5'), 4.01 (1H, pseudo-t, H3'), 3.73 (1H, d of d, H4'), 3.32 and 3.27 (each 3H, each s, each $\mathrm{CH}_{3} \mathrm{O}$ ), $2.87(1 \mathrm{H}, \mathrm{d}, \mathrm{H} 5 \mathrm{a}), 2.54$ (1H, d, H5b); ${ }^{2} \mathrm{~J} 5 \mathrm{a}-5 \mathrm{~b}=15.8,{ }^{3} \mathrm{~J} 7-7-\mathrm{OH}=4.4$ and $5.8,{ }^{3} \mathrm{~J} \mathrm{I}^{\prime}-2^{\prime}=4.0,{ }^{3} \mathrm{~J} 3^{\prime}-3^{\prime}-\mathrm{OH}=5.4,{ }^{3} \mathrm{~J} 4^{\prime}-5^{\prime}=7.2 \mathrm{~Hz} .{ }^{13} \mathrm{C}$ NMR ((CD $\left.)_{2} \mathrm{SO}\right) \delta 168.7(\mathrm{C} 4), 151.1(\mathrm{C} 2), 102.9\left(\mathrm{C} 5^{\prime}\right), 95.2$ (C6), $91.8(\mathrm{C} 7), 89.4\left(\mathrm{C} 1{ }^{\prime}\right), 87.8$ (C2'), 84.8 (C4'), 74.9 (C3'), 54.1 and 52.2 (two $\mathrm{CH}_{3} \mathrm{O}$ ), 34.2 (C5). Low-resolution FAB-mass spectrum, m/e $317.0\left(20 \%, \mathrm{MH}^{+}-\mathrm{H}_{2} \mathrm{O}\right), 309.0\left(100 \%, \mathrm{MH}^{+}-2 \mathrm{H}_{2} \mathrm{O}\right)$.

1-(5-Deoxy-5,5-dimethoxy- $\beta$-D-arabinofuranosyl)uracil-6-carboxaldehyde 7,O2'-cycli-chemiacetal (18). Compou-nd 17 in $\left(\mathrm{CD}_{3}\right)_{2} \mathrm{SO}$ solution was observed by ${ }^{1} \mathrm{H}$ NMR to slowly equilibrate to a quaternary mixture of hydrate 17 , its corresponding aldehyde, and the diastereomers of 18 . This process was inhibited by the presence of water and promoted by heating or desiccation with $4 \AA$ molecular sieves. Aldehyde: ${ }^{1} \mathrm{H}$ NMR $\left(\left(\mathrm{CD}_{3}\right)_{2} \mathrm{SO}\right) \delta 10.8\left(1 \mathrm{H}\right.$, br, $\mathrm{NH}$, exchanges with $\left.\mathrm{D}_{2} \mathrm{O}\right)$, $9.35(1 \mathrm{H}, \mathrm{s}, \mathrm{CHO}), 5.99\left(1 \mathrm{H}, \mathrm{d}, \mathrm{H} 1^{\prime}\right), 5.71\left(1 \mathrm{H}, \mathrm{d}, 3\right.$ '-OH, exchanges with $\left.\mathrm{D}_{2} \mathrm{O}\right), 4.65(1 \mathrm{H}, \mathrm{d}$, H2'), 4.42 (1H, d, H5'), 4.13 (1H, pseudo-t, H3'), 3.79 (1H, pseudo-t, H4'), 3.30 and 3.29 (each $3 \mathrm{H}$, each s, each $\left.\mathrm{CH}_{3} \mathrm{O}\right), 3.04(1 \mathrm{H}, \mathrm{d}, \mathrm{H} 5 \mathrm{a}), 2.85(1 \mathrm{H}, \mathrm{d}, \mathrm{H} 5 \mathrm{~b}) ;{ }^{2} \mathrm{~J} 5 \mathrm{a}-5 \mathrm{~b}=15.6,{ }^{3} \mathrm{~J} 1^{\prime}-2^{\prime}=3.8,{ }^{3} \mathrm{~J} 3^{\prime}-$ $3^{\prime}-\mathrm{OH}=5.5,{ }^{3} \mathrm{~J} 4^{\prime}-5^{\prime}=5.2 \mathrm{~Hz}$.

Thus, 18 was obtained (quantitative by ${ }^{1} \mathrm{H} \mathrm{NMR}$ ) by heating 17 in $\left(\mathrm{CD}_{3}\right)_{2} \mathrm{SO}$ solution at $45{ }^{\circ} \mathrm{C}$ for $6 \mathrm{~h}$ over $4 \AA$ molecular sieves. As had been found for $5 \mathrm{c},{ }^{6 \mathrm{~b}, \mathrm{c}}$ the ${ }^{1} \mathrm{H}$ NMR spectrum of 18 in $\left(\mathrm{CD}_{3}\right)_{2} \mathrm{SO}$ solution revealed the presence of a $3: 1$ mixture of the two 7,O2'-cyclic hemiacetal diastereomers. Major diastereomer: ${ }^{1} \mathrm{H}$ NMR $\left(\left(\mathrm{CD}_{3}\right)_{2} \mathrm{SO}\right) \delta 11.5(1 \mathrm{H}, \mathrm{bs}, \mathrm{NH}$, exchanges with $\left.\mathrm{D}_{2} \mathrm{O}\right), 7.63\left(1 \mathrm{H}\right.$, d, hemiacetal $\mathrm{OH}$, exchanges with $\left.\mathrm{D}_{2} \mathrm{O}\right), 5.77(1 \mathrm{H}, \mathrm{d}, 3 \mathrm{\prime}-\mathrm{OH}$, exchanges with $\left.\mathrm{D}_{2} \mathrm{O}\right), 5.67(1 \mathrm{H}, \mathrm{d}$, hemiacetal $\mathrm{CH}), 5.63\left(1 \mathrm{H}, \mathrm{d}, \mathrm{H} 1{ }^{\prime}\right), 5.62(1 \mathrm{H}, \mathrm{s}, \mathrm{H} 5), 4.39$ $\left(1 \mathrm{H}, \mathrm{d}, \mathrm{H} 2^{\prime}\right), 4.26\left(1 \mathrm{H}, \mathrm{d}, \mathrm{H} 5^{\prime}\right), 4.04\left(1 \mathrm{H}, \mathrm{d}, \mathrm{H} 3^{\prime}\right), 3.73\left(1 \mathrm{H}, \mathrm{d}, \mathrm{H} 4^{\prime}\right) ;{ }^{3} J 7-7-\mathrm{OH}=6.4,{ }^{3} \mathrm{~J} 1^{\prime}-2^{\prime}=2.6$, ${ }^{3} \mathrm{~J}^{\prime}-3^{\prime}-\mathrm{OH}=4.8,{ }^{3} \mathrm{~J} 4^{\prime}-5^{\prime}=7.6 \mathrm{~Hz} .{ }^{13} \mathrm{C} \mathrm{NMR}\left(\left(\mathrm{CD}_{3}\right)_{2} \mathrm{SO}\right) \delta 162.5(\mathrm{C} 4), 150.6$ and $148.8(\mathrm{C} 2 / \mathrm{C} 6)$, 102.9 (C5'), 99.5 (C5), 87.1 (C7), 84.2 (C4'), 78.0 (C1'), 75.4 (C3'), 73.8 (C2'), 54.2 and 54.8 (two $\left.\mathrm{CH}_{3} \mathrm{O}\right)$. Minor: ${ }^{1} \mathrm{H}$ NMR $\left(\left(\mathrm{CD}_{3}\right)_{2} \mathrm{SO}\right) \delta 11.5\left(1 \mathrm{H}\right.$, br, $\mathrm{NH}$, exchanges with $\left.\mathrm{D}_{2} \mathrm{O}\right), 7.92(1 \mathrm{H}$, $\mathrm{d}$, hemiacetal $\mathrm{OH}$, exchanges with $\left.\mathrm{D}_{2} \mathrm{O}\right), 5.78\left(1 \mathrm{H}, \mathrm{d}, 3^{\prime}-\mathrm{OH}\right.$, exchanges with $\left.\mathrm{D}_{2} \mathrm{O}\right), 5.76(1 \mathrm{H}, \mathrm{s}$, H5), $5.54\left(1 \mathrm{H}, \mathrm{d}, \mathrm{H} 1^{\prime}\right), 5.46(1 \mathrm{H}, \mathrm{d}$, hemiacetal $\mathrm{CH}), 4.32\left(1 \mathrm{H}, \mathrm{d}, \mathrm{H} 2^{\prime}\right), 4.23\left(1 \mathrm{H}, \mathrm{d}, \mathrm{H} 5^{\prime}\right), 4.04$ $\left(1 \mathrm{H}, \mathrm{d}, \mathrm{H} 33^{\prime}\right), 3.72\left(1 \mathrm{H}, \mathrm{d}, \mathrm{H} 4^{\prime}\right) ;{ }^{3} \mathrm{~J} 7-7-\mathrm{OH}=7.7,{ }^{3} \mathrm{~J} 1^{\prime}-2^{\prime}=2.4,{ }^{3} \mathrm{~J}^{\prime}-3^{\prime}-\mathrm{OH}=4.9,{ }^{3} \mathrm{~J} 4^{\prime}-5^{\prime}=8.0 \mathrm{~Hz}$. ${ }^{13} \mathrm{C}$ NMR ((CD $\left.)_{2} \mathrm{SO}\right) \delta 162.4(\mathrm{C} 4), 151.0$ and 150.8 (C2/C6), 102.9 (C5'), 98.1 (C5), 89.3 (C7), $84.3\left(\mathrm{C}^{\prime}\right), 78.6\left(\mathrm{C}^{\prime}\right), 77.7\left(\mathrm{C}^{\prime}\right), 75.4\left(\mathrm{C}^{\prime}\right), 54.0$ and 52.5 (two $\left.\mathrm{CH}_{3} \mathrm{O}\right)$ ). Low-resolution CI-mass spectrum, m/e $317.1\left(50 \%, \mathrm{MH}^{+}\right), 309.0\left(100 \%, \mathrm{MH}^{+}-\mathrm{CH}_{3} \mathrm{OH}\right)$. High-resolution CI-mass spectrum for $\mathrm{C}_{12} \mathrm{H}_{17} \mathrm{~N}_{2} \mathrm{O}_{8}\left(\mathrm{MH}^{+}\right)$: calcd 317.0985, found 317.0987 .

Deprotection of 18. Hydrolysis of the 5'-(dimethyl) acetal in $18(18.3 \mathrm{mg}, 0.058 \mathrm{mmol})$ with $50 \%$ aqueous TFA $(0.5 \mathrm{~mL})$ was sluggish $\left(23{ }^{\circ} \mathrm{C}, 4 \mathrm{~d}\right)$, but proceeded. The residue obtained upon rotary evaporation to dryness in vacuo was separated by ascending preparative chromatography (silica gel, $15 \% \mathrm{MeOH} / \mathrm{CH}_{2} \mathrm{Cl}_{2}$ as eluent), and the purified compound was dried (Abderhalden, $\left.\mathrm{P}_{2} \mathrm{O}_{5}, 45-50{ }^{\circ} \mathrm{C}\right)$. Lyophilization of an aqueous solution followed by desiccation gave the deprotected material $(8.4 \mathrm{mg}, 54 \%)$ found to exist as a $2: 1$ mixture of 7,O2'-cyclic hemiacetal 
diastereomers in $\left(\mathrm{CD}_{3}\right)_{2} \mathrm{SO}$ solution, by ${ }^{1} \mathrm{H}$ NMR. Major diastereomer: ${ }^{1} \mathrm{H}$ NMR $\left(\left(\mathrm{CD}_{3}\right)_{2} \mathrm{SO}\right)$ $\delta 11.6(1 \mathrm{H}, \mathrm{bs}, \mathrm{NH}), 9.41\left(1 \mathrm{H}, \mathrm{s}, 4^{\prime}-\mathrm{CHO}\right), 7.77(1 \mathrm{H}, \mathrm{d}, 7-\mathrm{OH}), 6.20\left(1 \mathrm{H}, \mathrm{d}, 3^{\prime}-\mathrm{OH}\right), 5.84(1 \mathrm{H}, \mathrm{d}$, H1'), 5.64 (1H, d, H7), 5.63 (1H, s, H5), 4.47 (1H, d, H2'), 4.30 (1H, s, H4'), 4.25 (1H, d, H3').

${ }^{13} \mathrm{C} \mathrm{NMR}\left(\left(\mathrm{CD}_{3}\right)_{2} \mathrm{SO}\right) \delta 201.4\left(\mathrm{C}^{\prime}\right), 158.1(\mathrm{C} 4), 150.7$ and 148.1 (C2/C6), 99.9 (C5), 89.2 (C7), 87.1 (C4'), 79.6 (C1'), 76.9 and $71.4\left(\mathrm{C}^{\prime} / \mathrm{C}^{\prime}\right)$; 3J7-7-OH $=6.5 \mathrm{~Hz}$. Minor: ${ }^{1} \mathrm{H}$ NMR $\left(\left(\mathrm{CD}_{3}\right)_{2} \mathrm{SO}\right)$ $\delta 11.6(1 \mathrm{H}, \mathrm{bs}, \mathrm{NH}), 9.42\left(1 \mathrm{H}, \mathrm{s}, 4^{\prime}-\mathrm{CHO}\right), 8.00(1 \mathrm{H}, \mathrm{d}, 7-\mathrm{OH}), 6.20(1 \mathrm{H}, \mathrm{d}, 3$ '-OH $), 5.79(1 \mathrm{H}, \mathrm{d}$, H1'), 5.60 (1H, s, H5), 5.50 (1H, d, H7), 4.37 (1H, d, H2'), 4.26 (1H, s, H4'), 4.24 (1H, d, H3'); $3 \mathrm{~J} 7-7-\mathrm{OH}=7.7 \mathrm{~Hz} .{ }^{13} \mathrm{C} \mathrm{NMR}\left(\left(\mathrm{CD}_{3}\right)_{2} \mathrm{SO}\right) \delta 201.6\left(\mathrm{C} 5{ }^{\prime}\right), 157.7(\mathrm{C} 4), 150.6$ and $148.3(\mathrm{C} 2 / \mathrm{C} 6)$, 98.3 (C5), 89.1 (C7), 89.0 (C4'), 79.9 (C1'), 77.2 and 75.9 (C2'/C3').

(5'-R)-1-(5-Methoxy- $\beta$-D-arabinofuranosyl)-6-(dihydroxymethyl)-7,O2':7,O5'-dianhydrouracil (6b). A solution of $18(20.0 \mathrm{mg})$ in a mixture of anhydrous DMA $(0.2 \mathrm{~mL})$ and anhydrous C6H6 $(0.4$ $\mathrm{mL}$ ) was treated with $\mathrm{pTsOH}$ monohydrate $(0.3 \mathrm{mg})$. The resultant mixture was heated at reflux for $24 \mathrm{~h}$ under argon and then was evaporated to dryness in vacuo. The residue was dissolved in a small amount of methanol and separated by ascending preparative chromatography twice (silica gel, $10 \% \mathrm{MeOH} / \mathrm{CH}_{2} \mathrm{Cl}_{2}$ as eluent) to give $6 \mathrm{~b}$ as a gum $(4.5 \mathrm{mg}, 25 \%) .{ }^{1} \mathrm{H} \mathrm{NMR}$ $\left(\left(\mathrm{CD}_{3}\right)_{2} \mathrm{SO}\right) \delta 11.5(1 \mathrm{H}, \mathrm{br}, \mathrm{NH}), 6.37\left(1 \mathrm{H}, \mathrm{d}, \mathrm{H} 1{ }^{\prime}\right), 5.83$ and 5.77 (each 1H, each s, H5 and H7), $5.71\left(1 \mathrm{H}, \mathrm{d}, 3^{\prime}-\mathrm{OH}\right), 4.93\left(1 \mathrm{H}, \mathrm{d}, \mathrm{H} 5^{\prime}\right), 4.67\left(1 \mathrm{H}, \mathrm{d}, \mathrm{H} 2{ }^{\prime}\right), 4.38\left(1 \mathrm{H}, \mathrm{d}, \mathrm{H} 3{ }^{\prime}\right), 4.21$ (1H, d, H4'), $3.23(3 \mathrm{H}, \mathrm{s}, \mathrm{CH} 3 \mathrm{O}) ;{ }^{3} \mathrm{~J} 1^{\prime}-2 \mathrm{2}^{\prime}=6.4,{ }^{3} \mathrm{~J} 3^{\prime}-3^{\prime}-\mathrm{OH}=4.3,{ }^{3} \mathrm{~J}^{\prime}-5^{\prime}=3.8 \mathrm{~Hz} .{ }^{13} \mathrm{C} \mathrm{NMR}\left(\left(\mathrm{CD}_{3}\right)_{2} \mathrm{SO}\right) \delta$ 162.4 (C4), 150.8 and 149.1 (C2/C6), 99.4 and 99.2 (C5'/C5), 88.0 (C7), 83.1 (C4'), 79.7 (C1'), 76.7 and $76.4\left(\mathrm{C}^{\prime} / \mathrm{C}^{\prime}\right), 55.3\left(\mathrm{CH}_{3} \mathrm{O}\right)$. Low-resolution FAB-mass spectrum, m/e $285.1(40 \%$, $\left.\mathrm{MH}^{+}\right)$. High-resolution FAB-mass spectrum for $\mathrm{C}_{11} \mathrm{H}_{13} \mathrm{~N}_{2} \mathrm{O}_{7}\left(\mathrm{MH}^{+}\right)$: calcd 285.0723, found 285.0722 .

\section{References}

${ }^{\dagger}$ This work was performed at Southern Illinois University where it was supported in part by grants from SIU's Office of Research Development and Administration. Current support from NIH (NIGMS56878) is gratefully acknowledged. This work has been communicated in brief, preliminary form: Groziak, M. P.; Lin, R. Nucleosides Nucleotides 1997, 16, 1419.

1. (a) Kittaka, A.; Kato, H.; Tanaka, H.; Nonaka, Y.; Amano, M.; Nakamura, K. T.; Miyasaka, T. Tetrahedron 1999, 55, 5319. (b) Wang, G.; Girardet, J.-L.; Gunic, E. Tetrahedron 1999, 55, 7707. (c) Marquez, V. E.; Ezzitouni, A.; Russ, P.; Siddiqui, M. A.; Ford, H.; Feldman, R. J.; Mitsuya, H.; George, C.; Barchi, J. J. J. Am. Chem. Soc. 1998, 120, 2780. (d) Björsne, M.; Szabó, T.; Samuelsson, B.; Classon, B. Bioorg. Med. Chem. 1995, 3, 397. (e) Nielsen, P.; Pfundheller, H. M.; Wengel, J. J. Chem. Soc., Chem. Commun. 1997, 825. (f) Bar, N. C.; Patra, R.; Achari, B.; Mandal, S. B. Tetrahedron 1997, 53, 4727. (g) Steffens, R.; Leumann, C. J. J. Am. Chem. Soc. 1997, 119, 11548. (h) Bolli, M.; Lubini, P.; Leumann, C. Helv. Chim. Acta 1995, 78, 2077. (i) V. Tetrahedron Lett. 1995, 36, 7375. (j) Rao, S. N. Nucleosides Nucleotides 1995, 14, 1179. (k) Megati, S.; Ealick, S. E.; Naguib, F. N. M.; el Kouni, M. H.; Klein, R. S.; Otter, B. A. Nucleosides Nucleotides 1994, 13, 2151. (1) Plavec, J.; Tong, W.; Chattopadhyaya, J. J. Am. Chem. Soc. 1993, 115, 9734. (m) Chemla, P. Tetrahedron Lett. 1993, 46, 7391. (n) Hsu, L.-H.; Wise, D. S.; Kucera, L. S.; Drach, J. C.; Townsend, L. B. J. Org. Chem. 1992, 57, 3354. (o) Koole, L. H.; Wu, J.-C.; Neidle, S.; Chattopadhyaya, J. J. Am. Chem. Soc. 1992, 114, 2687. (p) Otter, B. A.; Patil, S. A.; Spada, 
M. R.; Jelicks, L. A.; Yoshimura, Y.; Matsuda, A.; Klein, R. S. Nucleosides Nucleotides 1992, 11, 615. (q) Hsu, L.-Y.; Wise, D. S.; Drach, J. C.; Townsend, L. B. Chin. Pharm. J. 1991, 43, 275. (r) Ueda, T. Nucleosides Nucleotides 1985, 4, 67. (s) Kameyama, K.; Sako, M.; Hirota, K.; Maki, Y. J. Chem. Soc., Chem. Commun. 1984, 1658.

2. (a) Maruyama, T.; Kimura, S.; Sato, Y.; Honjo, M. Chem. Pharm. Bull. 1986, 34, 3623.

3. (b) Maruyama, T.; Kimura, S.; Sato, Y.; Honjo, M. J. Org. Chem. 1983, 48, 2719. (c) Kumadaki, I.; Nakazawa, M.; Kobayashi, Y. Maruyama, T.; Honjo, M. Tetrahedron Lett. 1983, 24, 1055.

4. (a) Voznyi, Ya. V.; Kochetkov, N. K. Carbohydr. Res. 1977, 54, 300. (b) Bochkov, A. F.; Chernetskii, V. N.; Kochetkov, N. K. Carbohydr. Res. 1975, 43, 35. (c) Bochkov, A. F.; Dashunin, V. M.; Shashkov, A. S.; Kochetkov, N. K. Izv. Akad. Nauk SSSR, Ser. Khim. 1975, 1061. (d) Bochkov, A. F.; Chernetskii, V. N.; Kochetkov, N. K. Izv. Akad. Nauk SSSR, Ser. Khim. 1975, 465. (e) Bochkov, A. F.; Obruchnikov, I. V.; Chernetskii, V. N.; Kochetkov, N. K. Carbohydr. Res. 1974, 36, 191.

5. Bertolini, M.; Glaudemans, C. P. J. Carbohydr. Res. 1971, 18, 131.

6. See reference $1 \mathrm{k}$ for the comment that "...the majority of cyclonucleosides suffer from electronic and/or conformational shortcomings that make them less than ideal probes."

7. (a) Groziak, M. P.; Koohang, A. J. Org. Chem. 1992, 57, 940. (b) Groziak, M. P.; Koohang, A.; Stevens, W. C.; Robinson, P. D. J. Org. Chem. 1993, 58, 4054. (c) Groziak,

8. M. P.; Lin, R.; Stevens, W. C.; Wotring, L. L.; Townsend, L. B.; Balzarini, J.; Witvrouw, M.; De Clercq, E. Nucleosides Nucleotides 1996, 15, 1041.

9. (a) Myers, A. G.; Gin, D. Y.; Rogers, D. H. J. Am. Chem. Soc. 1994, 116, 4697. (b) Ueda,

10. T. In Chemistry of Nucleosides and Nucleotides; Townsend, L. B., Ed.; Plenum: New York, 1988; Vol.1, pp 1-112; and related references cited therein. (c) Jones, G. H.; Taniguchi, M.; Tegg, D.; Moffatt, J. G. J. Org. Chem. 1979, 44, 1309. (d) Jones, G. H.; Moffatt, J. G. J. Am. Chem. Soc. 1968, 90, 5337.

11. Hayakawa, H.; Haraguchi, K.; Tanaka, H.; Miyasaka, T. Chem. Pharm. Bull. 1987, 35, 72.

12. (a) Simmons, H. E.; Wiley, D. W. J. Am. Chem. Soc. 1960, 82, 2288. (b) Newkome, G. R.; Sauer, J. D.; McClure, G. L. Tetrahedron Lett. 1973, 18, 1599.

13. A similar nucleoside 1,2-epoxide reportedly forms by the action of $m$-CPBA on a D-erythropent-1-enofuranosyluracil: Kittaka, A.; Tanaka, H.; Miyasaka, T.; Yamaguchi, K. Nucleosides Nucleotides 1992, 11, 37.

14. Watson, S. C.; Eastham, J. F. J. Organomet. Chem. 1967, 9, 165.

15. Dess, D. B.; Martin, J. C. J. Org. Chem. 1983, 48, 4155.

16. Groziak, M. P.; Lin, R.; Robinson, P. D. Acta Crystallogr. 1995, C51, 1204. 